\title{
Novos rumos para disseminação e recuperação da informação científica em saúde coletiva.
}

\section{New directions for dissemination and recovery of scientific information in collective health.}

\section{Nuevas direcciones de difusión y recuperación de información científica en salud colectiva.}

Julio Cesar CABRAL ${ }^{1}$

Ana Valéria Machado MENDONÇA ${ }^{2}$

\begin{abstract}
RESUMO: Trata-se de uma investigação nos processos de publicização da informação científica em Saúde Coletiva. Diante da dinâmica de informacionalização da Internet, cujo volume de conteúdos gerados não tem precedentes, certamente a disseminação e a recuperação das informações tornam-se mais difíceis e imprecisas; neste cenário em que o pesquisador não consegue acompanhar o volume de informação disponibilizado, a busca e recuperação da informação é um desafio. Objetivou-se observar que ações as editoras científicas em Saúde Coletiva estão adotando para tratar esta problemática.Observou-se entre as Editoras a adoção de novas tecnologias da informação e comunicação, periódicos eletrônicos, Open Access, a adoção da prática de união de interesses, por meio de consórcios institucionais e o uso sistemas interoperantes. Com uma abordagem quantitativa, baseia-se em uma observação sobre uma base amostral de sítios de editoras científicas, estratificadas pelo WebQualisda CAPES em A1; A2; B1; B2; B3; B4; B5 e C por área do conhecimento de Saúde Coletiva.
\end{abstract}

Palavras-chave:Saúde Coletiva, Informação e Comunicação em Saúde, Tecnologia da Informação.

ABSTRACT: This is an investigation in publicizing processes of scientific information in public health. On the dynamics of the Internet informationalization, whose volume generated content is unprecedented, certainly dissemination and retrieval of information become more difficult and inaccurate; in this scenario where the researcher can not keep up with the volume of available information, the search and retrieval of information is a challenge. The objective was to observe what actions scientific publishers in Public Health are taking to address this problem. It was observed among the publishers to adopt new information technologies and communication, electronic journals, Open Access, the adoption of the practice of union interests through institutional consortia and the use of interoperable systems. With a quantitative approach, based on an observation on a

1 Mestre em Saúde Coletiva pela Universidade de Brasília (UnB); especialista em Educação e Promoção em Saúde pela UnB; graduação em Sistemas de Informação; Perito Forense em Sistemas Comprometidos - Escola Superior de Redes. E-mail: jcesar@unb.br

2 Professora adjunta do Departamento de Saúde Coletiva da Universidade de Brasília (UnB). Coordenadora do Mestrado Profissionalizante e do Núcleo de Estudos em Saúde Pública da UnB.E-mail: valeriamendonca@unb.br 
sample basis of sites of scientific publishers, stratified by CAPES WebQualis in A1; A2; B1; B2; B3; B4; B5 and C area by the Public Health knowledge.

Keywords: Public Health, Information and Communication in Health Information Technology.

RESUMEN: Se trata de una investigación en la difusión de los procesos de información científica en salud pública. Em la dinámica de la informatización de Internet, cuyo volumen contenido generado no tiene precedentes, sindudaladifusión y recuperación de la información cada vez más difícil e imprecisa; en este es cenario donde el investigador no puedemantenerse al díaconelvolumen de informacióndisponible, la búsqueda y recuperación de lainformación es undesafío. El objetivo era observar qué acciones editoriales científicas enSalud Pública están tomando para hacer frente a este problema. Se observó entre los editores para adoptar nuevas tecnologías de la información y la comunicación, revistas electrónicas, acceso abierto, la adopción de la práctica de los intereses del sindicato a través de consórcios institucionales y el uso de los sistemas interoperables. Conun enfoque cuantitativo, basado en una nota sobre una base de muestreo a lossitios de los editores científicos, estratificados por CAPES WebQualisen A1; A2; B1; B2; B3; B4; Área B5 y C por el conocimiento de la Salud Pública.

Palabras clave:Salud Pública, Información y la Comunicación em la Tecnología de la Información de la Salud.

\section{INTRODUÇÃO}

Definida como a troca de informações entre membros da comunidade científica, a comunicação científica inclui atividades associadas à produção, à disseminação e ao uso da informação, o que tem sido, historicamente, condição essencial ao pensamento científico (1). Atualmente esta troca tem sido dificultado pelo elevado volume de informações disponibilizadas na Internet que vem forçando novos hábitos de absorção desta produção científica, e mudando profundamente comportamentos individuais, exigindo dos pesquisadores novas visões e maneiras de fazer ciência (2). Somando-se à situação, a globalização, cujo

\footnotetext{
"significado mais profundo transmitido é o de caráter indeterminado, indisciplinado e de autopropulsão dos assuntos mundiais; a ausência de um centro, de um painel de controle, de uma comissão diretora, de um gabinete administrativo"(3).
}

Tudo converge na Explosão Informacional, que segundo Branco (4), é um fenômeno problemático na Internet, caracterizada por mudanças políticas, tecnológicas e organizacionais.

À medida que a internet se torna a soma de conglomerados, novos modelos de divulgação científica vão surgindo; quanto mais dados são armazenados na internet, mais difícil torna-se encontrar algo específico (5). Para aqueles que necessitam estar imersos em dados de pesquisa, descritores, sons, imagens e inúmeros outros formatos de informações, o excesso de informação pode causar um resultado adverso à necessidade em curso. Desse modo, faz-se necessário proporcionar maior eficiência na disseminação e recuperação, dando confiabilidade à pesquisa 
mediada por tecnologias de informação e comunicação (5).

Observa-se também que nas últimas décadas a Internet alterou não apenas a dinâmica do fluxo da comunicação científica, mas também o modo de fazer ciência, com a integração da comunidade científica com outros setores da sociedade, atuando em redes transdisciplinares e heterogêneas de colaboração entre instituições de natureza variada. A evolução dessas redes de colaboração foi facilitada pelos avanços dos meios de comunicação e da Internet.

O processo de produção do conhecimento científico passou a ser não-linear, com participação de todos os interessados, desde o momento da concepção das pesquisas até a aplicação de seus resultados, trazendo consequências tanto para as etapas de redação como de validação. Esta última, antes restrita à comunidade científica, passa também a ser realizada pela comunidade em geral, que pode verificar a confiabilidade dos resultados e as implicações sociais dos avanços de pesquisas (6).

\section{PERIÓDICOS CIENTÍFICOS ELETRÔNICOS}

O periódico científico, como principal canal de comunicação formal, tem vivenciado transformações no decorrer das últimas três décadas, das quais passou de publicações impressas para títulos desenvolvidos para ambiente eletrônicos. Originaram-se das newsletters e das redes de conferências eletrônicas há cerca de 30 anos. A primeira concepção de um periódico eletrônico surgiu na década de 70, por Sondak e Schwartz, que propunham ofornecimento de arquivos que pudessem ser lidos por computadores para as bibliotecas e através de microfichas para assinantes individuais (7).

Para Oliveira(2008), a definição de periódico eletrônico apresenta as mesmas diversidades encontradas na conceituação de publicação eletrônica, sendo apresentadas pelos autores de forma bastante diferenciada:

- "publicação cujo meio primário de envio para assinantes é através de arquivo de computador" (BOMBAK et al., 1992, citado por CHAN, 1999, p. 10);

- "publicação eletrônica com texto completo, que pode incluir imagens, e pretende ser publicado indefinidamente" (UNIVERSITY, 1994, citado por CHAN, 1999, p. 11);

- "periódico criado para o meio eletrônico e disponível apenas nesse formato" (LANCASTER, 1995, p. 520);

- "periódicos acadêmicos que são disponibilizados através da Internet e suas tecnologias associadas" (HARRISON; STEPHEN, 1995, p. 593);

- "aquele que possui artigos com texto integral, disponibilizados via rede, com acesso on-line, e que pode ou não existir em versão impressa ou em qualquer outro tipo de suporte" (CRUZ et al., 2003, p. $48)$;

Atualmente existem mais de 1,8 milhões de registros International Standard Serial Number(ISSN) com um crescimento anual de 60 a 100 mil registros anuais (8).

Observa-se na tabela 1 que o formato impresso cresceu 27,31\% no período de 2004 a 2009, enquanto que a versão eletrônica cresceu $77,49 \%$ no mesmo período. 
Tabela 1 - Números de registros de periódicos por suporte.

\begin{tabular}{|l|l|l|l|l|l|l|l|l|l|}
\hline & 2004 & 2005 & 2006 & 2007 & 2008 & 2009 & 2010 & 2011 & 2012 \\
\hline Printed & 1124235 & 1182241 & 1230056 & 1280680 & 1332581 & 1395463 & 1447705 & 1500130 & 1546700 \\
Online & 25451 & 32967 & 41448 & 50353 & 62004 & 72337 & 83507 & 97563 & 113092 \\
\hline
\end{tabular}

Fonte: ISSN International Centre - http://www.issn.org/wp-content/uploads/2013/07/number_of_records_medium. pdf

\section{ACESSO ABERTO À INFORMAÇÃO CIENTÍFICA}

Em 2013 aumentaram as manifestações de questionamentos dos meios de comunicação da pesquisa em consequência das inovações, reestruturações e soluções em curso que apontam para o acesso aberto, a publicação continuada de artigos no lugar de fascículos, o uso de ferramentas das redes sociais para a gestão e disseminação de informação, novas métricas para medir influência, uso e impacto dos artigos, etc (9).

O movimento do acesso aberto surgiu da crise dos custos das publicações impressas no início dos anos 2000 e das oportunidades de acesso oferecidas pela publicação digital na Internet, e hoje se encontra plenamente consolidado para os periódicos em formato digital. Estudo recente encomendado pela Comissão Europeia concluiu que quase $50 \%$ dos artigos publicados entre 2004 e 2011 nos países da Comunidade, Estados Unidos, Canadá, Japão e Brasil encontram-se disponíveis em acesso aberto na Internet em abril de 2013. O Brasil lidera com 63\% dos artigos em acesso aberto em grande parte devido ao Scientific Electronic Library Online (SciELO). De fato, de acordo com o Directory of Open Access Journals(DOAJ), o Brasil está situado em segundo lugar, com 921 periódicos, atrás apenas dos Estados Unidos, com 1021 periódicos.

Cientes da importância do conhecimento gerado pelas universidades e do acesso aos conteúdos por elas produzidos, o Governo, o Senado Federal brasileiro têm desenvolvido movimentos políticos paralelos que trabalham frente à necessidade de disseminação da produção científica nacional, trata-se de Ciência Aberta, publicada em revistas de livre acesso. Em uma perspectiva mais ampla, o Acesso Aberto favorece a educação e o uso da informação científica pelo público. No Brasil, um projeto de lei Acesso Livre (PL 1120/2007) dispõe sobre o processo de disseminação da produção técnico-científica de ensino superior que obriga as instituições públicas a construírem repositórios institucionais para depósito do inteiro teor da produção, como o projeto Scientific Electronic Library Online (SciELO)Brasil, iniciativa da BIREME, implantada em 1998 (10).

Outras ações que vale destacar parte do Instituto (IBICT) que em setembro de 2005, lançou um manifesto de apoio a este movimento que consta uma série de recomendações, aos diversos segmentos da comunidade científica; submissão, aprovação e desenvolvimento do Projeto de Publicações Periódicas de Acesso Livre (PCAL) junto à Financiadora de Estudos e Projetos do Ministério da Ciência e Tecnologia (FINEP); prospecção, identificação, absorção, customização, 
aperfeiçoamento e distribuição de tecnologias para o tratamento e disseminação da informação, que dão suporte às ações de acesso livre, como: 1) o software para construção e gestão de revistas científicas Sistema Eletrônica de Editoração de Revistas (SEER); e 2) Eprints software para construção de repositórios institucionais e temáticos; e 3) Capacitação de técnicos para o uso e desenvolvimento dessas tecnologias no Brasil, que ao longo dos anos já capacitou mais de mil técnicos e criou mais de 530 publicações científicas eletrônicas de acesso livre (11).

A tendência é que o número das publicações em Acesso Aberto siga aumentando, mas discretamente, uma vez que pesquisadores estão tentados por vários motivos e às vezes forçados a preferir o modelo tradicional de periódicos por assinatura e de alto impacto. Em contrapartida, políticas públicas de um número crescente de países e de agências de fomento à pesquisa determinam que a publicação de resultados de pesquisa financiada com recursos públicos seja em Acesso Aberto (12).

A pesquisa em plataformas abertas em qualquer lugar pode ser um exemplo dessa inovação. Trazendo mais níveis de complexidade no processamento da informação, estas dificuldades residem justamente no alinhamento de suas economias e sociedades num novo contexto socioeconômico, caracterizado pela sociedade da informação. Este desalinhamento entre as diferentes regiões do globo e internas de cada país é agravado pelas mudanças no fluxo da produção do conhecimento científico e, sobretudo, devido às alterações ocorridas com as publicações científicas eletrônicas, contribuindo para o fenômeno de infoexclusão ${ }^{4}(13)$.

Gráfico 1- O desenvolvimento da publicação Acesso Aberto 1993-2009.

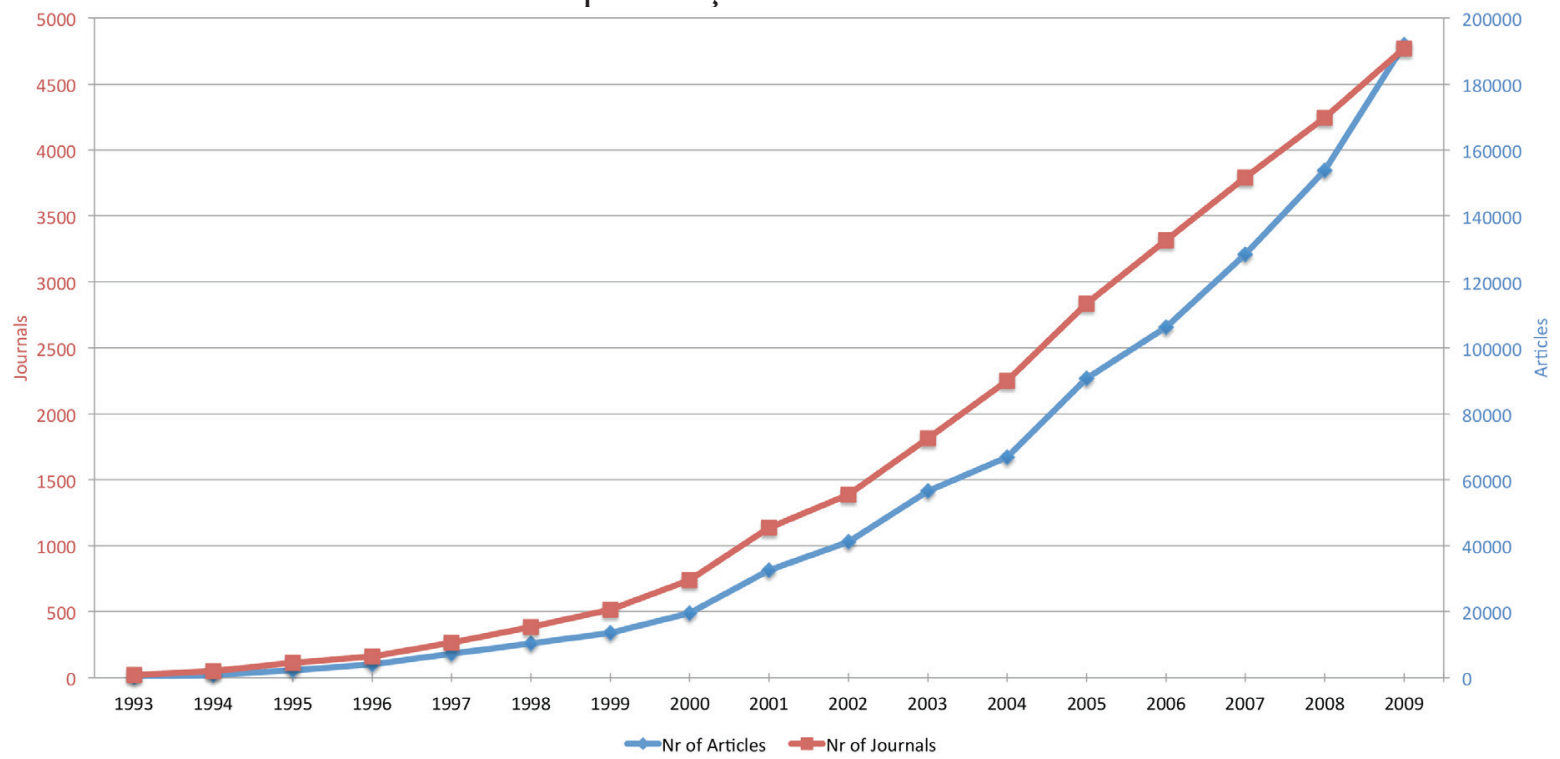

Fonte:The Development of Open Access Laakso M, Welling P, Bukvova H, Nyman L, Björk B-C, et al. 2011

O gráfico 1 mostra a evoluçãodo número derevistas e artigos de pesquisa em Acesso Aberto

4 Desconhecimento que origina falta ou impossibilidade de acesso a informação, .notadamente através das novas tecnologias de comunicação como a Internet."infoexclusão", in Dicionário Priberam da Língua Portuguesa [em linha], 2008-2013, http://www.priberam.pt/dlpo/infoexclus\%C3\%A3o [consultado em 28-08-2015]. 
publicados durante o período de1993-2009. Nele, observa-se gradual crescimento, evidenciando a sustentabilidade do Acesso Aberto como uma forma de publicação científica.

\section{CONSÓRCIOS INSTITUCIONAIS}

A adoção da prática de união de interesses por meio de consórcio de bibliotecas para a seleção, aquisição, manutenção e preservação da informação eletrônica, apresenta-se como uma das formas encontradas pelas instituições e profissionais envolvidos com o objetivo de diminuir ou dividir os custos orçamentários, ampliar o universo de informações disponíveis aos usuários e o sucesso dessas atividades (14).

Considerando que o uso das tecnologias de informação será tanto mais intensivo quanto maior for a capacidade da sociedade em assimilá-las, a sociedade brasileira parece caminhar, simultaneamente, para uma descoberta desse novo meio, assimilando cada vez mais informações e utilizando-se das redes de informação numa cadeia crescente (15).

Esta prática pode ser observada com a consolidação do Consórcio Nacional de Periódicos Eletrônicos Coordenação de Aperfeiçoamento de Pessoal de Nível Superior (CAPES), criado originalmente no início dos anos 90 e que reúne conteúdos científicos e disponibiliza à comunidade acadêmico-científica brasileira, objetivando oferecer ao seu público: a) facilidade de acesso à informação científica, reunindo em um única espaço virtual publicações do mundo; b) acesso ao conhecimento atualizado, os artigos, livros e patentes recentemente publicados nos Estados Unidos, Ásia e Europa podem ser recuperados em tempo real; c) inserção internacional do conhecimento científicos, onde o pesquisador tem acesso à produção dos autores, periódicos e sociedades internacionais conceituadas; e sobretudo d) democratização do acesso à informação, onde um pesquisador vinculado tem acesso ao mesmo conhecimento compartilhado para todos os outros membros da rede, tratando-se de uma maior capilaridade.

Outro exemplo de consórcio, o Centro Latino-Americano e do Caribe de Informação em Ciências da Saúde, também conhecido pelo seu nome original Biblioteca Regional de Medicina (BIREME), é um centro especializado da Organização Pan-Americana da Saúde / Organização Mundial da Saúde (OPAS/OMS), orientado à cooperação técnica em informação científica em saúde.

Observa-se no gráfico 2 uma evolução de portal consorciado da CAPES, com um crescimento de 411,11\% no números de instituições no período de 2003 a 2012. 
Gráfico 2 - Evolução do número de instituições com acesso ao Portal de Periódicos - 2002 2012.

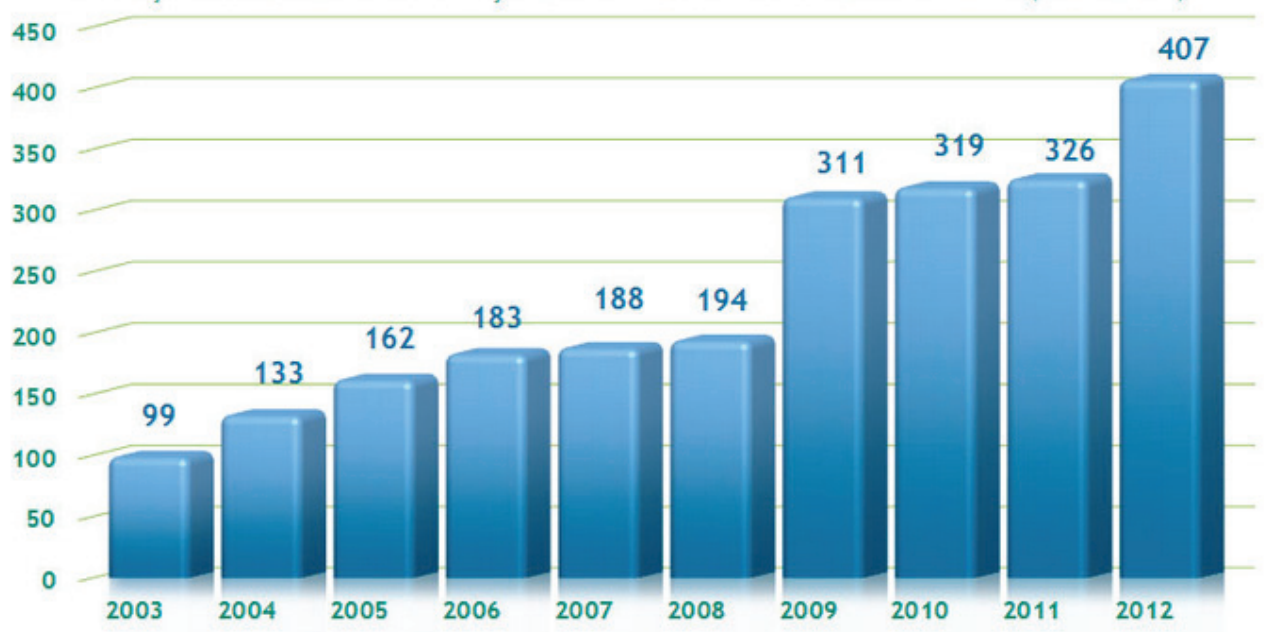

Fonte: Portal de Periódicos CAPES, 2010.

Vale citar outros casos de consórcios em saúde, externos, e outras áreas de conhecimento, como a canadense Canadiam Research Knowledge Network, programa sem fins lucrativos das universidades canadenses, dedicado a expandir a pesquisa acadêmica em formato digital para continuar o trabalho desenvolvido pelo Canadiam National Site Licenave Ensing Project iniciado em 2000 e consorciando 73 instituições de ensino e pesquisa.

\section{SISTEMAS INTEROPERANTES E NORMATIZAÇÕES}

Sob a égide da tecnologia da informação, a principal característica da sociedade é a informação, muito mais que outros insumos como a energia, máquinas e artefatos (13). Trata-se de uma matériaprima, pois são tecnologias para agir sobre a informação, e não apenas informação para agir sobre as tecnologias(12). Atualmente ocorre uma transição tão importante quanto a que o mundo assistiu ao ver a passagem do texto manuscrito para o impresso, o advento das novas Tecnologias da Informação e Comunicação(TIC) e as constantes mudanças nos processos e inovações tecnológicas, estão fazendo com que instituições repensem o modo de produção, acesso e disseminação da informação (14).

A interoperabilidade assenta-se na ideia de cooperação e requer o estabelecimento de políticas da informação que ofereçam condições para viabilizar a produção tecnológica e a recepção da informação científica e tecnológica, além de garantias de condições tecnológicas para que o processo ocorra(17).

Para que haja interoperabilidade torna-se necessário a padronização das informações, facilitando a troca de dados, sua reutilização ao longo do tempo e também ajuda a prevenir aprisionamento a ferramentas e formatos proprietários. Mas para um padrão ser bem sucedido, é necessário que 
seja usado por todos os agentes envolvidos nas transações. Os ambientes, em geral, promovem a integração dos dados e dos recursos, mas sem a necessária organização sintática e semântica de seu acervo, os formatos são diversos e o crescimento de dispositivos móveis que podem capturar estes dados. Cria-se um ambiente heterogêneo, onde é necessária a discussão profunda da interoperabilidade de dados e recursos(18).

O Portal de Revistas Científicas da BVS registra cercade 8.600 revistas científicas correntes da área da saúde, das quais 50\% estão disponíveis em formato eletrônico. Das 5.236 revistas indexadas na baseMedical Literature Analysis and Retrieval System Online(MEDLINE) e registradas no Portal da BVS, 3.457 (66\%)estão em formato eletrônico, em acesso aberto ou controlado. No caso das revistas publicadas em países daAmérica Latina e Caribe e indexadas na base de dados LILACS, a percentagem de revistas eletrônicas passou de 18\% em 2001 para 78\% em 2006.

\section{PERCURSO METODOLÓGICO}

A pesquisa realizou uma seleção do WebQualis ${ }^{5}$ feita por consulta por Critérios Qualis por Área - Saúde Coletiva, com isso, obteve-se um arquivo Portable Document Format (PDF) contendo relatório Classificação/Área Avaliação listando o ISSN, Título, Estrato, Área de Avaliação e Status, que informa a situação atual, Atualizado ou Em Avaliação, do periódico na data da consulta.

Em seguida, foi desenvolvida a base amostral daclassificação de periódicosem A1; A2; B1; B2; B3; B4;B5 e C WebQualis CAPES.Para a determinação do tamanho da amostra considerou-se o número total de 2.601 periódicos. O erro amostral adotado foi de $5 \%$ e nível de confiança de $95 \%$, resultando numa amostra de 335 periódicos em a partir de:

$$
n=\frac{N \cdot Z^{2} \cdot p \cdot(1-p)}{Z^{2} \cdot p \cdot(1-p)+e^{2} \cdot(N-1)}
$$

Por considerar importante a inclusão de números representativos de todos os estratos, optou-se por realizar uma amostra estratificada simples em oito deles (A1, A2 B1, B2, B3, B4, B5 e C) com uma fração amostral equivalente a n/N, onde $n=335$ e $N=2.601$.Os dados abaixo são dispensáveis pois você pode sintetizar o cálculo e ir direto ao ponto.

Assim, $\mathrm{n} / \mathrm{N}=0,129$.

Então,

$$
\mathrm{n}_{1}=\mathrm{n} / \mathrm{N}^{*} \mathrm{~N}_{1}=18(18+2)
$$

$\mathrm{n}_{2}=\mathrm{n} / \mathrm{N}^{*} \mathrm{~N}_{2}=35(35+3)$

$\mathrm{n}_{3}=\mathrm{n} / \mathrm{N}^{*} \mathrm{~N}_{3}=62(62+6)$

5 Aplicativo desenvolvido pela CAPES que estratifica a qualidade da produção intelectual dos programas de pós-graduação. Disponível em http://qualis.capes.gov.br/webqualis/principal.seam Acessado em: 23/Jul/2013. 


$$
\begin{aligned}
& \mathrm{n}_{4}=\mathrm{n} / \mathrm{N}^{*} \mathrm{~N}_{4}=68(68+7) \\
& \mathrm{n}_{5}=\mathrm{n} / \mathrm{N}^{*} \mathrm{~N}_{5}=35(35+3) \\
& \mathrm{n}_{6}=\mathrm{n} / \mathrm{N}^{*} \mathrm{~N}_{5}=49(49+5) \\
& \mathrm{n}_{7}=\mathrm{n} / \mathrm{N}^{*} \mathrm{~N}_{5}=19(19+2) \\
& \mathrm{n}_{8}=\mathrm{n} / \mathrm{N}^{*} \mathrm{~N}_{5}=49(49+5)
\end{aligned}
$$

Ao final, optou-se por incluir $10 \%$ a mais do valor calculado por estrato para compensar as possíveis perdas. Após a definição da quantidade de periódicos por estrato, realizou-se a seleção aleatória simples sem reposição em cada estrato utilizando o pacote estatístico Statistical Package for the Social Sciences(SPSS).

A Tabela 2 apresenta o universo de periódicos científicos em Saúde Coletiva elencados pelo WebQualis da CAPES, observados durante a pesquisa.

\begin{tabular}{lll}
\hline PERIÓDICOS EM SAÚDE COLETIVA \\
\hline Estrato & N de título & $\%$ \\
\hline A1 & 140 & $5,39 \%$ \\
A2 & 272 & $10,46 \%$ \\
B1 & 478 & $18,37 \%$ \\
B2 & 524 & $20,14 \%$ \\
B3 & 275 & $10,58 \%$ \\
B4 & 383 & $14,72 \%$ \\
B5 & 145 & $5,57 \%$ \\
C & 384 & $14,77 \%$ \\
\hline TOTAL & 2601 & $100,00 \%$ \\
\hline
\end{tabular}

Tabela 2 - Base Amostral - Números de periódicos em Saúde Coletiva estratificadas WebQualis $-2007 / 2009,2010,2011$ e 2012.

\section{MATRIZ DE DADOS E ÁRVORE DE DECISÃO}

Com uma abordagem quantitativa, este estudo trabalha com um esquema classificatório que permite registrar os resultados das buscas observacionais. Estabeleceu-se uma Matriz de Dados, desenvolvida em Microsoft Office Excel 2007 cujos recursos incluem uma interface intuitiva e capacitadas ferramentas de cálculo e de construção de gráficos. Estabelecida a Matriz de Dados, usou-se o software Weka para criação do Modelo de Decisão.

Quanto à árvore de decisão, trata-se de uma estrutura em árvore hierárquica, usada para tomar decisões baseadas em questões sobre as informações disponíveis. Pode ser utilizada para classificação 
de dados, predição de saídas e geração de regras de classificação de fácil compreensão e nas mais diversas áreas do conhecimento por apresentarem poucas restrições quanto às características das variáveis adotadas, de tal modo que não exige distribuição normal, além de admitir a dependência entre as variáveis (19).

Considerada uma técnica de mineração de dados cuja motivação consiste em descobrir conhecimento a partir de uma base de dados, a árvore de decisão é capaz de converter o conhecimento em regras; para isso é necessário que seja feito o particionamento ou classificação, objetivando responder qual dos atributos (variáveis constantes na base de dados) será o nó raiz(17). Sua função precípua consiste em particionar recursivamente um conjunto de treinamento, de modo que cada subconjunto obtido apresente casos de uma única classe.

\section{RESULTADOS E DISCUSSÃO}

Consolidando o universo da pesquisa, a tabela 3 traz um panorama dos periódicos científicos em Saúde Coletiva estratificados pelo WebQualis, a partir da qual se observa que a maior frequência está estratificada como B2, peso 50 de qualidade segundo a Qualis CAPES, indexadas na Base Scopus com índice $\mathrm{H}$ entre os percentis 45 ratificação e 75 ou revistas indexadas no SciELO, com indicador de impacto entre os percentis 50 e 70; e a menor frequência, A1 - peso 100 - Revistas indexadas na base Scopus com índice H acima do percentil 95 ou Journal Citation Reports(JCR) com fator de impacto acima de 4,0 no casodo periódico não estar no Scopus.

\begin{tabular}{|l|l|l|}
\hline Qualis CAPES & Frequência $=346$ & $\%$ \\
\hline A1 & 20 & 5,8 \\
\hline A2 & 38 & 11,0 \\
\hline B1 & 67 & 19,4 \\
\hline B2 & 73 & 21,1 \\
\hline B3 & 38 & 11,0 \\
\hline B4 & 43 & 12,4 \\
\hline B5 & 21 & 6,1 \\
\hline C & 46 & 13,3 \\
\hline
\end{tabular}

Tabela 3-Frequências dos periódicos científicos em Saúde Coletiva estratificados pelo WebQualis Fonte: CABRAL. JC. 2014.

\section{Panorama dos periódicos científicos que possuem instrumentos de buscas multi-base}

Vale ressaltar que a interoperabilidade acontece quando as organizações trabalham com seus aplicativos e com suas informações para um único objetivo, ou seja, uma única invocação de pesquisa aciona inúmeros sistemas e bases de dados em busca de uma informação específica. Assim, podese afirmar que as ferramentas de buscas avançadas multibase podem interoperar, assim podemos considerar multibase como disponíveis em locais e formatos iguais ou diferentes, e.g. ambientes virtuais de aprendizagem, repositórios institucionais e revistas em sistemas diferentes. 
Neste cenário observou-se que dos periódicos científicos eletrônicos cujos instrumentos de busca sejam avançados multibase, $71,0 \%$ tem a temporalidade maior que 20 anos de publicização, $89,1 \%$ usam o idioma Inglês como língua para publicação e 87,0\% adotam políticas Open Access. Destacam-se a forma de publicização, periódicos que usam Editora para publicização de seus artigos, alcançam $97,8 \%$ de possibilidade de interoperar com outras bases institucionais, enquanto que periódicos científicos que usam sítio próprios alcançam $0,7 \%$ e os que usam OJS, $0,0 \%$ de probabilidade de interoperar.

\begin{tabular}{ll}
\hline QUALIS & \% que interoperam \\
\hline A1 & 70 \\
A2 & 60,52 \\
B1 & 49,25 \\
B2 & 64,38 \\
B3 & 34,21 \\
B4 & 18,60 \\
B5 & 0 \\
C & 0
\end{tabular}

Tabela 4 - Relação periódicos em Saúde Coletiva que possuem ferramentas de buscas multibase. Fonte:CABRAL, JC.

O tabela 4 traz um panorama dos periódicos científicos em Saúde Coletiva que possuem, através de consorciamento, recursos tecnológicos interoperantes. Observa-se que a oferta do recurso diminui conforme a estratificação CAPES diminui, observando uma variação nos periódicos B2, com uma representatividade de 21,1\% entre os periódicos científicos em Saúde Coletiva.

\section{CONSIDERAÇÕES FINAIS}

Vaidhyanathan (2011) evidencia em seu trabalho que o elevado volume de informação disponibilizados na Internet vem causando mudanças comportamentais, forçando novos hábitos e exigindo dos pesquisadores novas visões e maneiras de fazer ciência, assim, esta pesquisa alinha-se e se propôs conhecer e analisar os atuais processos para disseminação da informação científica. Avanços nos usos das tecnologias de informação e comunicação têm sido vivenciados no decorrer das últimas décadas, conforme apontam Oliveira (2008), Packer (2013), Kuramoto (2008) e Krzyzniwski (1998). Complementam-se aos avanços das tecnologias da informação e comunicação, movimentos institucionais e sociais como consorciamento de editoras científicas e o movimento de Acesso Aberto à Informação Científica, respectivamente. Seguindo Amorim e Verguerio (2006), a análise da Base Amostral evidenciou para o consorciamentoinstitucinal como uma da formas encontradas pelas instituições de ampliar a disseminação informação.

Evidenciou-se que paralelamente ao crescimento da Internet, houve mudanças associadas, e.g., convergência de novas tecnologias da informação e comunicação; aumento na publicização e visibilidade de artigos; crescimento nos números de periódicos científicos, organizações e 
consolidações de consórcios institucionais e universitários; e sobretudo mudanças no volume e fluxo informacional, consequentemente mudanças na forma de se fazer ciência, acredita-se positivas.

No decorrer da pesquisa evidenciou-se que paralelamente aos avanços das tecnologias de informação e comunicação, a comunicação científica seguiu na mesma velocidade nos avanços na publicização, ou seja, impactos na visibilidade. Isto aponta um panorama positivo sobre tendências de evoluções maiores na disseminação e apropriação do conhecimento científico. Observou-se um crescimento na oferta de periódicos científicos nos formatos eletrônicos sobre o impresso, com tendência de consolidar-se, devido a dinamicidade, abrangência e outros benefícios.

Para além de conhecer os recursos tecnológicos utilizados pelas editoras científicas em Saúde Coletiva, como padrões e normatizações; compreender, diante da explosão informacional, seus movimentos para tratamento publicizição científica, i.e, organizações em rede. Com isso, esta investigação buscou conhecer, ações que favoreçam a interoperabilidade de seus sistemas de publicização, visando disseminar e recuperar de forma eficiente o conhecimento científico.

Com este exponencial avanço nos meios de comunicação, demonstrou-se a necessidade de instituições organizarem-se em portais consorciados, Consórcios Institucionais, i.g, Portal da CAPES. Os dados analisados mostraram que em todo mundo, várias instituições vêm adotando instrumentos e alinhando tecnologias que focam a interoperabilidade; essas ações evidenciam soluções bem sucedidas diante do cenário dinâmico.

Percebem-se avanços em termo de Cooperação na Área da Saúde, entretanto, especificamente na subárea de Saúde Coletiva precisa estreitar laços, consociar e normatizar, vale ressaltar que o sucesso de padrões e normatizações ocorre a partir da sua universalidade, ampla aceitação, entretanto, sua aplicabilidade não cabe em todos os cenários institucionais, o que não impede alterações e ajustes tecnológicos, enfim, somar mais esforços para interoperarem.

Com um impacto positivo, a interoperabilidade consolida-se, influenciando diretamente o modo como as pessoas produzem e trocam informações no âmbito pessoal, profissional e acadêmico.

Os assuntos tratados neste artigo apontam que as tecnologias emergentes trazem boas perspectivas para a comunidade científica, promovendo acesso equitativo às informações. Apontam também que, isoladamente, as Editoras Científicas em Saúde Coletiva não possuem recursos tecnológicos, operacionais e humanos para soluções na disseminação ordenada de seus conteúdos, e diante do cenário dinâmico da Internet, a solução chega através de consorciamento científico.

\section{RERÊNCIAS BIBLIOGRÁFICAS}

1- FERREIRA, MMP; TARGINO, MG; Acessibilidade e visibilidade de revistas científicas eletrônicas, São Paulo. Editora SENAC. 2010.

2- VAIDHYANATHAN, S. A googlelização de tudo, $1^{\text {a }}$ edição, 2011. 
3- BAUMAN, Z. Globalização: as conseqüências humanas. Rio de Janeiro. Jorge Zahar Editora. 1999.

4-BRANCO, MAF. Informação e Saúde - Uma ciência e suas políticas em uma nova era/ Informationandhealth: a scienceand its politics in a new age. Rio de Janeiro, FIOCRUZ; 2006.

5- FRAGOSO, S; RECUERO, R; AMARAL, A. Métodos dePesquisa para internet. Porto Alegre: Sulina. 2011.

6- CASTRO, RCF. Impacto da Internet no fluxo da comunicação científica em Saúde. Rev Saúde Pública. 2006.

7 -OLIVEIRA, EBPM. Periódicos Científicos Eletrônicos: definições e histórico. Inf. \&Soc.:Est., João Pessoa, v.18, n.2, p. 69-77, maio/ago. 2008.

8 - CIEPS [Internet]: International Centre for the Registration of Serial - International Serial Number. Disponível em: http://www.issn.org/. Acessado em 22/09/2105.

9 - PACKER, A. Rumo à inovação e renovação da gestão dos periódicos SciELO. SciELO em Perspectiva. Conferência SciELO 15 Anos. Disponível em: http://blog.scielo.org/ blog/2013/12/18/rumo-a-inovacao-e-renovacao-da-gestao-dos-periodicos-scielo/. Dez. 2013.

10 - Projetos de Leis e Outras Proposições PL 1120/ 2007 - Acesso livrehttp://www.camara. gov.br/proposicoesWeb/fichadetramitacao?idProposicao=352237 11 - KURAMOTO, H. Acesso livre à informação científica: novos desafios. Linc em Revista, Rio de Janeiro; 2008.

12. Quanto custa publicar em acesso aberto?. SciELO em Perspectiva. Disponível em: http:// blog.scielo.org/blog/2013/09/18/quanto-custa-publicar-em-acesso-aberto//

13- AMORIM, AM. A globalização do mercado de periódicos científicos eletrônicos e os consórcios de bibliotecas universitárias brasileiras: desafios à democratização do conhecimento científico. Dissertação de Ciência da Informação e Documentação. Escola de Comunicação e Artes da Universidade de São Paulo. São Paulo; 2002.

14-KRZYZANIWSKI, RF. Biblioteca eletrônica de revistas científicas internacionais: Projeto de consórcio. Ci. Inf. v.27 n.2 Brasília; 1998. Disponível em: http://dx.doi.org/10.1590/S010019651998000200009 .

15- AMORIM, Antonio M.; VERGUEIRO, Waldomiro. Consórcios de bibliotecas no Brasil: um desafio à democratização do conhecimento. Perspectivas em Ciência da Informação, v. 11, n. $1 ; 2006$.

16- ELUAN, AA, Análise dos usos da plataforma Open Journal System para o processo de editoração eletrônica: Um estudo focado nos editores de periódicos científicos eletrônicos de acesso livre em Ciência da Informação e Biblioteconomia no Brasil. Dissertação (Mestrado Ciência da Informação) - Universidade Federal de Santa Catarina, Programa de Pós-Graduação em ciência da Informação. Florianópolis; 2009.

17- MOREIRA, W; LARA, MLG, Ontologias, categorias e interoperabilidade semântica. Revista da Informação V 13 n 4 agosto; 2012. 
18- MUCHERONI, ML, SILVA, JFM. A, 2011. Interoperabilidade dos sistemas de informação sob o enfoque da análise sintática e semântica de dados na web. Ponto de Acesso, V5 n1, p03-18. Abril 2011. UFBA.

19 - SOARES, RAS. Modelo de suporte à decisão aplicado ao atendimentodas vítimas de acidentes de trânsitona cidade de João Pessoa. Universidade Federal da Paraíba. Centro de Ciências Exatas e da Natureza. Departamento de Estatística. Mestrado em Modelos de Decisão e Saúde; 2012.

Artigo apresentado em 10-01-15

Artigo aprovado em 20-07-15

Artigo publicado no sistema em 26-09-15 